\title{
ALE STRESS UPDATE FOR TRANSIENT AND QUASISTATIC PROCESSES
}

\author{
ANTONIO RODRÍGUEZ-FERRAN ${ }^{1}$, FOLCO CASADEI ${ }^{2}$ AND ANTONIO HUERTA ${ }^{1, *}$ \\ ${ }^{1}$ Departamento de Matemática Aplicada III, E.T.S. de Ingenieros de Caminos, Universitat Politècnica de Catalunya, \\ Campus Nord C-2, E-08034 Barcelona, Spain \\ ${ }^{2}$ Structural Mechanics Unit, Institute for Systems, Informatics and Safety, \\ Joint Research Centre of the European Commission, I-21020 Ispra (Va), Italy
}

\begin{abstract}
A key issue in Arbitrary Lagrangian-Eulerian (ALE) non-linear solid mechanics is the correct treatment of the convection terms in the constitutive equation. These convection terms, which reflect the relative motion between the finite element mesh and the material, are found for both transient and quasistatic ALE analyses. It is shown in this paper that the same explicit algorithms can be employed to handle the convection terms of the constitutive equation for both types of analyses. The most attractive consequence of this fact is that a quasistatic simulation can be upgraded from Updated Lagrangian (UL) to ALE without significant extra computational cost. These ideas are illustrated by means of two numerical examples.
\end{abstract}

KEY WORDS: arbitrary Lagrangian-Eulerian formulation; non-linear solid mechanics; stress update; explicit algorithm

\section{INTRODUCTION}

The Arbitrary Lagrangian-Eulerian (ALE) formulation of continuum mechanics was initially developed in the context of fluid dynamics (see for instance Reference 1 and references cited therein), with the goal of overcoming the limitations of the Lagrangian and Eulerian formulations. The basic idea is to allow the computational mesh to move in an arbitrary manner, independent of material motion. By doing so, it is possible to avoid the excessive mesh distortion of a purely Lagrangian formulation or the cumbersome boundary tracking of a purely Eulerian description. Various explicit algorithms can be found in the literature for the ALE remeshing (i.e. selection of mesh velocity), see References $2-5$.

More recently, the ALE formulation has been extended to non-linear solid mechanics. ${ }^{6-10,3}$ Compared to ALE fluid dynamics, the main additional difficulty is then the stress update (i.e. the time integration of the constitutive equation). Indeed, the rate-form constitutive equation of ALE non-linear solid mechanics contains a convective term that accounts for the relative motion between mesh and material. Because of this, the stress update cannot be performed at the Gauss-point level, as simply as in a Lagrangian analysis, and more involved procedures are required. In fact, the correct treatment of the convective term in the constitutive equation is a key point in ALE non-linear solid mechanics.

\footnotetext{
* Correspondence to: Antonio Huerta, Departamento de Matemática Aplicada III, Universitat Politecnica de Catalunya, Campus Nord C-2, E-08034 Barcelona, Spain. E-mail: huerta@etseccpb.upc.es
} 
If a fractional-step method is chosen, the material and convective terms of the governing equations are treated separately. ${ }^{11}$ Each time step consists of two phases: a material phase and a convection phase. The basic goal of this paper is to show that the convection phase of the stress update can be performed with the same explicit algorithms for ALE transient analysis (where an explicit time integration for the momentum balance is typically employed) and ALE quasistatic analysis (where implicit time marching is commonly chosen). There is consequently no need to develop specific algorithms for each type of analysis.

An important consequence of this fact is that a quasistatic analysis can be upgraded from an Updated Lagrangian (UL) formulation to an ALE description at (near) zero extra computational cost, because the additional features of the ALE computation with respect to the UL computation (convection phase of the stress update and remeshing) can be performed by means of explicit algorithms, which have a negligible computational cost in the context of implicit time stepping.

The situation is different for transient dynamic analysis, where explicit time marching is often preferred. With respect to an UL computation, the extra computational cost per time step of an ALE computation is no longer negligible. It must be recalled, however, that the ALE formulation allows for larger time steps than the purely Lagrangian formulation, see References 11 and 12 and this can have a crucia

An outline of this paper is non-linear solid mechanics

between transient and qua the context of fractionalstress undate are presented and disun
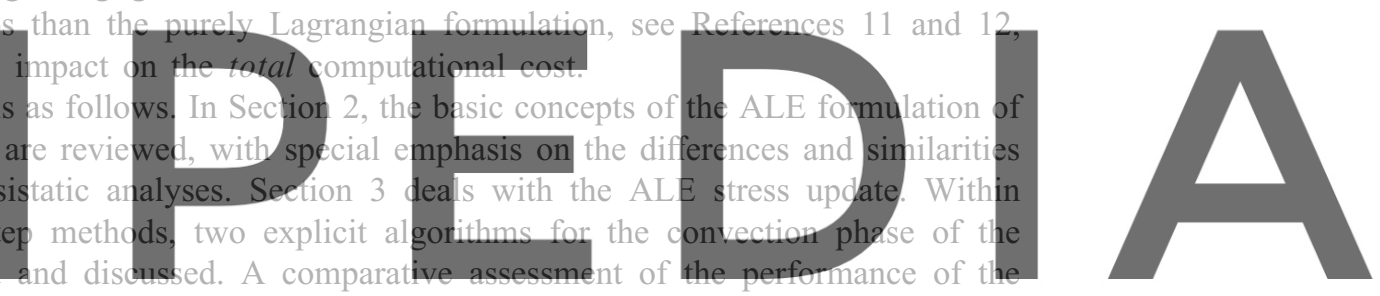

algorithms, both for transient and quasistatic processes, can be found in Section 4. Finally, some

\section{BASIC ALE EQUATIONS}

A detailed presentation of the ALE formulation of continuum mechanics may be found in Reference 1. The specific issues of ALE non-linear solid mechanics are addressed in Reference 11. Only the basic concepts and the notation that will be used throughout the paper are presented here.

\subsection{ALE kinematics}

Two domains are commonly employed in continuum mechanics: the material domain $R_{X}$, made up of material particles $\mathbf{X}$, and the spatial domain $R_{x}$, consisting of spatial points $\mathbf{x}$. Neither of these two is taken as the reference in the ALE description, so a third domain is needed: the referential domain $R_{\chi}$, formed by reference (or grid) points $\chi$.

One-to-one transformations between the three domains are required. A convenient way to provide them is by means of the equations of material motion $\mathbf{x}=\mathbf{x}(\mathbf{X}, t)$, and mesh motion $\mathbf{x}=\mathbf{x}(\chi, t)$, which yield the spatial position $\mathbf{x}$ of a material particle $\mathbf{X}$ and a grid point $\chi$, respectively, at time $t$.

If the instant $t_{0}$ is taken as a reference, material displacements $\mathbf{u}$ and mesh displacements $\widehat{\mathbf{u}}$ can be, respectively, defined as $\mathbf{u}(\mathbf{X}, t)=\mathbf{x}(\mathbf{X}, t)-\mathbf{x}\left(\mathbf{X}, t_{0}\right)$ and $\widehat{\mathbf{u}}(\chi, t)=\mathbf{x}(\boldsymbol{\chi}, t)-\mathbf{x}\left(\boldsymbol{\chi}, t_{0}\right)$.

In a similar fashion, material velocity $\mathbf{v}$ and mesh velocity $\widehat{\mathbf{v}}$ are obtained, respectively, by differentiating the equations of material motion and mesh motion presented previously with respect 
to time while keeping the particle $\mathbf{X}$ or the grid point $\chi$ fixed:

$$
\mathbf{v}=\left.\frac{\partial \mathbf{x}(\mathbf{X}, t)}{\partial t}\right|_{\mathbf{X}}, \quad \widehat{\mathbf{v}}=\left.\frac{\partial \mathbf{x}(\chi, t)}{\partial t}\right|_{\chi}
$$

In Equations $(1)$, the notation $\left.\right|_{*}$ means 'holding $*$ fixed'. The link between material and mesh motion is provided by convective velocity $\mathbf{c}$

$$
\mathbf{c}=\mathbf{v}-\widehat{\mathbf{v}}
$$

that is, the velocity of material particles relative to grid points.

Mesh acceleration plays no role in the ALE formulation, so only the material acceleration a is needed, which may be expressed in the Lagrangian, Eulerian or ALE formulation, respectively, as

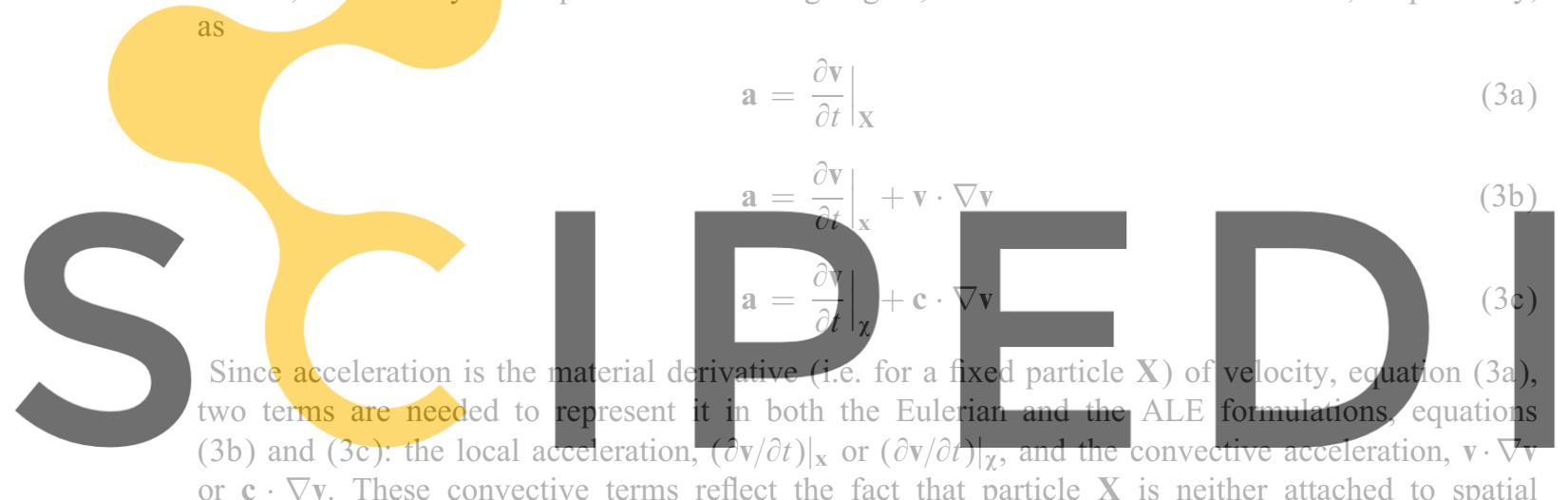

or $\mathbf{c} \cdot \nabla_{\mathbf{v}}$. These convective terms reflect the fact that particle $\mathbf{X}$ is neither attached to spatial

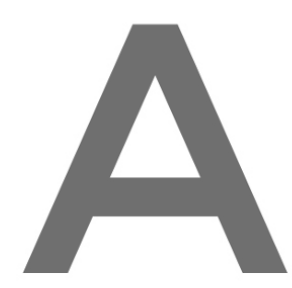

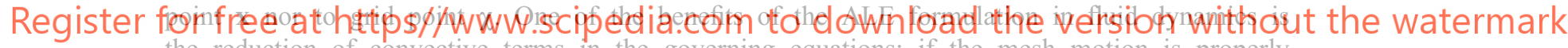

the reduction of convective terms in the governing equations: if the mesh motion is properly

selected, the convective velocity $\mathbf{c}$ is smaller than the material velocity $\mathbf{v}$, equation (2), so the

ALE convective acceleration $\mathbf{c} \cdot \nabla \mathbf{v}$ is smaller than the Eulerian convective acceleration $\mathbf{v} \cdot \nabla \mathbf{v}$.

Equation (3c) is in fact a particular case of the general relationship between material time derivatives and referential time derivatives

$$
\left.\frac{\partial f}{\partial t}\right|_{\mathbf{X}}=\left.\frac{\partial f}{\partial t}\right|_{\chi}+c_{i} \frac{\partial f}{\partial x_{i}}
$$

where $f$ is any scalar function. ${ }^{1,11}$

\subsection{ALE transient analysis}

\subsubsection{Conservation laws}

Equation (4) is the starting point to deduce the three fundamental conservation laws of continuum mechanics (mass, momentum and energy) in the ALE description. If mechanical effects are uncoupled from thermal effects, then the mass and momentum equations can be solved independently from the energy equation. The ALE version of these two equations is ${ }^{1}$

Balance of mass:

$$
\left.\frac{\partial \rho}{\partial t}\right|_{\chi}+c_{j} \frac{\partial \rho}{\partial x_{j}}=-\rho \frac{\partial v_{j}}{\partial x_{j}}
$$


Balance of momentum:

$$
\left.\left(\rho a_{i}=\right) \rho \frac{\partial v_{i}}{\partial t}\right|_{\chi}+\rho c_{j} \frac{\partial v_{i}}{\partial x_{j}}=\frac{\partial \sigma_{i j}}{\partial x_{j}}+b_{i}
$$

where $\rho$ is the density, $\boldsymbol{\sigma}$ is the Cauchy stress tensor and $\mathbf{b}$ is the force per unit volume.

A standard procedure in non-linear solid mechanics consists of dropping the equation of mass balance (5a), which is not explicitly accounted for, thus solving only the momentum balance (5b). In solid mechanics, the domain boundary is typically composed of material surfaces. Since these surfaces are accurately tracked by the Lagrangian description commonly employed in solid mechanics (and also by the ALE description, as commented later), the balance of mass is verified at the global level without explicitly stating it.

Of course, equation (5a) must also hold at the local level. A common assumption is to take the density $\rho$ constant. The mass balance then becomes

$$
\frac{\partial v_{j}}{\partial x_{j}}=0
$$

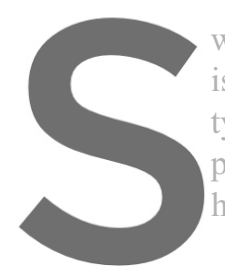

which is the well-known

is also commonly neglecte

typically induce very sma

preserving. ${ }^{13}$ That means

holds to sufficient approxi

In conclusion, if the two commo a a
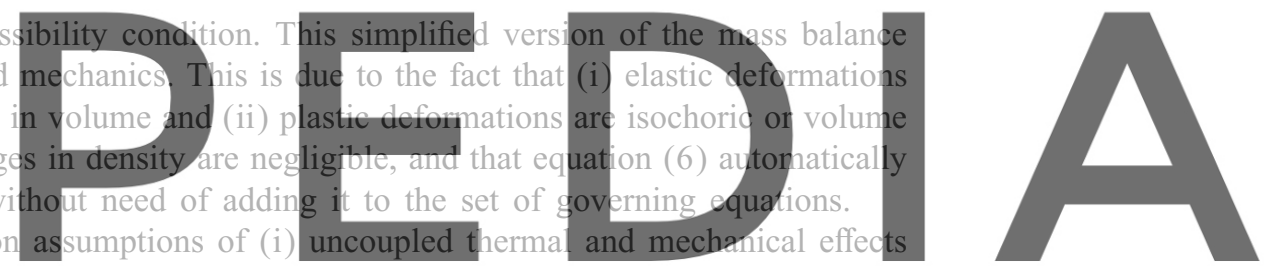

and (ii) constant density are made, then the only conservation law that needs to be solved is the

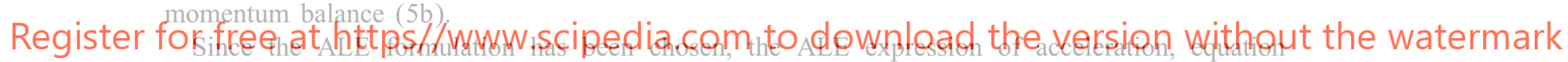

(3c), is employed to represent the inertia forces $\rho$ a (which are taken into account in the general, i.e. transient, case). Because of this, equation (5b) contains convective terms associated to the convective velocity c. These convective terms are similar to those encountered in the Eulerian formulation and reflect the relative motion between mesh and material.

Equation (5b) must be complemented with appropriate boundary conditions, which are imposed on the spatial domain. A detailed discussion of the implementation of boundary conditions in the ALE formulation can be found in References 14 and 12.

\subsubsection{Constitutive equations for non-linear solid mechanics}

In non-linear solid mechanics, material behaviour is often described by a rate-form constitutive equation, relating an objective rate of Cauchy stress to stress and stretching (rate-of-deformation), see Reference 15. In the ALE context, referential time derivatives, not material time derivatives, are employed to represent evolution in time. Specializing the general relationship (4) to the stress tensor, the ALE non-linear constitutive equation can be written as ${ }^{12}$

$$
\left.\frac{\partial \boldsymbol{\sigma}}{\partial t}\right|_{\chi}+c_{j} \frac{\partial \boldsymbol{\sigma}}{\partial x_{j}}=\mathbf{q}
$$

The left-hand-side of equation (7) is the material rate of stress $\left.(\partial \boldsymbol{\sigma} / \partial t)\right|_{\mathbf{x}}$ expressed in ALE format. The right-hand side $\mathbf{q}$ accounts for both the pure straining of the material and the rotational terms that counteract the non-objectivity of the material stress rate, Reference 12 . In the context of this 
Box 1. ALE transient analysis

$$
\begin{gathered}
\left.\rho \frac{\partial v_{i}}{\partial t}\right|_{\chi}+\rho c_{j} \frac{\partial v_{i}}{\partial x_{j}}=\frac{\partial \sigma_{i j}}{\partial x_{j}}+b_{i} \\
\left.\frac{\partial \boldsymbol{\sigma}}{\partial t}\right|_{\chi}+c_{j} \frac{\partial \boldsymbol{\sigma}}{\partial x_{j}}=\mathbf{q}
\end{gathered}
$$

paper, the most relevant feature of equation (7) is the convective term, which, as discussed for the momentum balance, reflects the motion of material particles relative to the mesh.

\subsubsection{Remeshing}

To define completely the problem posed by equations (5b) and (7), it is necessary to take equation (2) into account. Since the mesh moves independently from the material, it is necessary

to specify the mesh velocity $\widehat{\mathbf{v}}$ so that the convective velocity $\mathrm{c}$ can be determined.
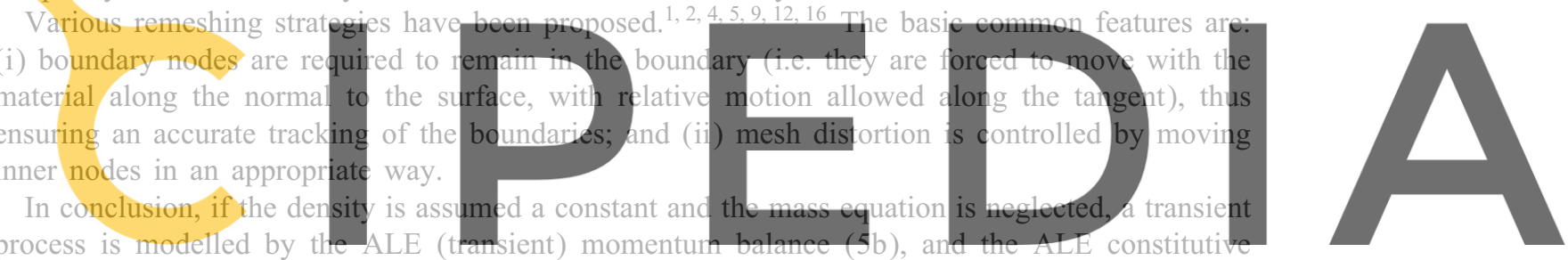

equation (7) (see Box 1) complemented with boundary conditions and a remeshing strategy to

\subsection{ALE quasistatic analysis}

In the previous section the ALE transient analysis has been discussed and the fundamental equations presented. It is very common, however, to do other types of analysis, such as steady state or quasistatic. In this section the quasistatic analysis is discussed and the equations are developed. Moreover, the steady state in an ALE context is also analysed to show that it lacks physical meaning.

A process is termed quasistatic if the inertia forces $\rho \mathbf{a}$ are negligible with respect to the other forces in the momentum equation (symbolically: $\rho \mathbf{a} \ll \nabla \cdot \boldsymbol{\sigma}+\mathbf{b}$ ). Inertia forces are negligible for physical reasons, so this must be independent of the kinematic description used. Consequently, one must also have

$$
\rho \mathbf{a}=\left.\rho \frac{\partial \mathbf{v}}{\partial t}\right|_{\mathbf{X}}=\rho\left(\left.\frac{\partial \mathbf{v}}{\partial t}\right|_{\mathbf{x}}+\mathbf{v} \cdot \nabla \mathbf{v}\right)=\rho\left(\left.\frac{\partial \mathbf{v}}{\partial t}\right|_{\chi}+\mathbf{c} \cdot \nabla \mathbf{v}\right) \ll \nabla \cdot \boldsymbol{\sigma}+\mathbf{b}
$$

which is independent of the arbitrarily chosen mesh velocity, and thus of the convection velocity.

Remark 1. Assuming negligible inertia forces does not imply zero accelerations (constant particle velocities). A process may have relevant, non-null local, convective and total accelerations and still be correctly modelled as quasistatic, if stress variations and/or body forces are much larger than inertia forces. This is a common situation in solid mechanics. See, for instance, Reference 12, where 
A. RODRÍGUEZ-FERRAN, F. CASADEI AND A. HUERTA

Box 2. ALE quasistatic analysis

$$
\begin{gathered}
\frac{\partial \sigma_{i j}}{\partial x_{j}}+b_{i}=0 \\
\left.\frac{\partial \boldsymbol{\sigma}}{\partial t}\right|_{\chi}+c_{j} \frac{\partial \boldsymbol{\sigma}}{\partial x_{j}}=\mathbf{q}
\end{gathered}
$$

the influence of the inertia forces is evaluated in two metal forming examples, or Reference 17

for other examples.

In quasistatic analysis, the momentum balance becomes

$$
\frac{\partial \sigma_{i j}}{\partial x_{j}}+b_{i}=0
$$

which is a static equilibrium equation where time and velocity play no role. That is, there are no convective terms in the ALE momentum balance for quasistatic processes.

The hypothesis of quasistatic process only concerns the momentum equation

on the constitutive equation. Thus, convective terms are present in the AI $E$

for quasistatic processes.

On the other hand, it is

the partial derivatives wit

and, theoretically, the sane concept

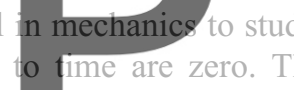

analysis would imply that $\partial \cdot /\left.\partial t\right|_{\chi}=0$. Thus, the momentum and constitutive equations are

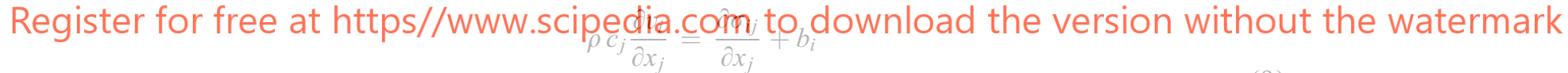$$
c_{j} \frac{\partial \sigma}{\partial x_{j}}=\mathbf{q}
$$

But a steady solution of equation (9) will not verify the same equation if the mesh velocity, $\widehat{\mathbf{v}}$ (i.e. the convection velocity c), is arbitrarily modified. It is important to recall that, on the one hand, the essence of ALE is the arbitrary nature of the mesh velocity (thus the convection), and, on the other, a steady process is dependent on the kinematic formulation employed. Therefore, in spite of Reference 10 , since the mesh velocity, $\widehat{\mathbf{v}}$, is arbitrary the ALE steady process is physically meaningless!

To conclude, a quasistatic process is modelled by the ALE (quasistatic) momentum balance (8), the ALE constitutive equation (7) (see Box 2) complemented with boundary conditions and the definition of mesh velocity.

\section{ALE STRESS UPDATE}

\subsection{Preliminaries}

It can be seen in Boxes 1 and 2 that the ALE momentum balance for transient processes, equation (5b), and for quasistatic processes, equation (8), are different: the former accounts for 
inertia forces, while the latter does not (a similar situation is found in the Lagrangian analysis of transient and quasistatic processes). Because of this, radically different techniques are employed for its time integration. The transient momentum balance is typically handled, both in the Lagrangian and ALE formulations, by an explicit, velocity-based scheme, ${ }^{18,19}$ where stability requirements impose a tight upper bound on the time step $\Delta t$. The quasistatic momentum balance, on the other hand, is commonly time-integrated with an implicit, incremental-iterative, displacement-based algorithm, which allows for larger time steps.

Regarding the ALE constitutive equation (7), it is valid for both transient and quasistatic processes (see Boxes 1 and 2). As previously remarked, the convective term in this equation is needed to represent relative motion between mesh and material, an essential feature of the ALE formulation.

Remark 2. The only difference in the ALE constitutive equation for transient and quasistatic cases lies in the meaning of variable $t$. In a transient analysis, $t$ represents physical time, which appears explicitly in the momentum balance (Box 1). For quasistatic analyses, however, physical time is usually not employed as an independent variable, because it is not present in the momentum balance (Box 2). Thus, the variable $t$ in the constitutive equation should be interpreted as the non-physical, pseudo-time prong Since the constitutive equation do schemes employed for the reasonable to demand that th update is needed to be cons implicit stress update procedure to
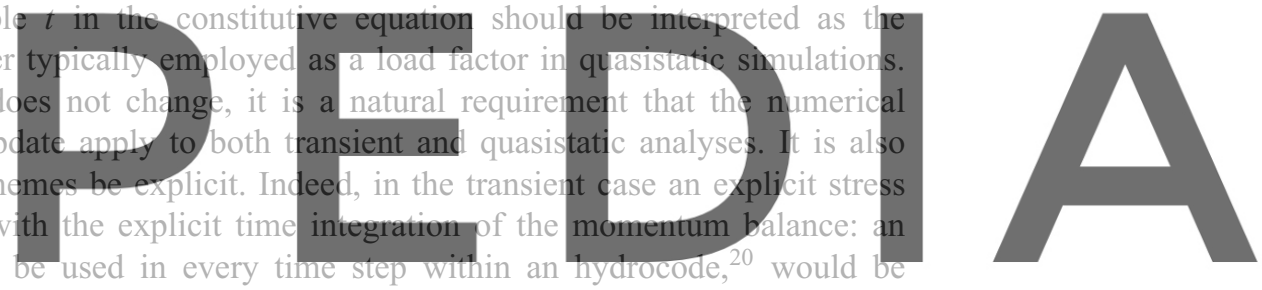
completely unaffordable. As for the quasistatic case, the stress update should be cheap-which, in

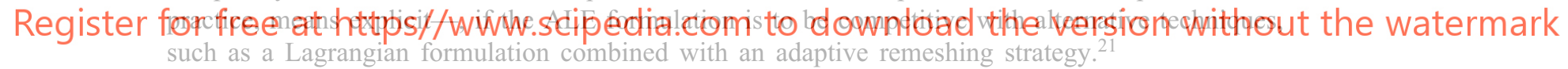

\subsection{Fractional-step methods}

Two different approaches are possible when dealing with the equations in Boxes 1 and 2: (i) solving the fully coupled equations, accounting for the various terms simultaneously or (ii) employing a fractional-step method, to treat material and convective effects separately. This second strategy will be used here.

In the ALE context, the coupled approach or unsplit approach has been adopted in References 8 and 22, among others. After finite element spatial semi-discretization, equations (5b) and (7) are transformed into matrix equations which contain convection terms for momentum and stress, respectively. These equations are then integrated in time by means of an explicit predictor-corrector method. The main advantage of the coupled approach is that there is no need to split the original equations.

If the second approach is taken, see References 7, 3 and 23, every time step is subdivided into two phases: a material (or Lagrangian) phase, where convective effects in the momentum balance (transient process) and constitutive equation are neglected, and a convection (or transport) phase. This operator split is commonly employed in the numerical integration of differential equations. ${ }^{24}$ In exchange for a certain loss of accuracy (with respect to the coupled approach), it offers a generic benefit: simpler equations means simpler, more robust algorithms, specifically designed for each equation. Regarding the ALE equations, an added advantage results: profit is taken from 
the experience in solid mechanics and fluid dynamics in handling material and convective terms, respectively.

The simplification associated to a fractional-step approach is especially interesting for the ALE constitutive equation. Indeed, the stress field $\boldsymbol{\sigma}$ is typically discontinuous across element edges, so its gradient cannot be reliably computed at the element level. Moreover, stress values are known at the Gauss points - where they are needed to compute internal forces-, not at the nodes. These two difficulties are easier to circumvent with a splitting technique.

This splitting technique may be regarded as the natural choice for ALE quasistatic analyses: since the momentum balance has no convective term (Box 2) it is completely handled by the material phase, so the transport phase need only deal with the convective term in the constitutive equation. The implementation of ALE capabilities in a Lagrangian code for quasistatic analysis is then a straightforward matter. ${ }^{25}$

With any of the two approaches, all the known numerical difficulties associated to convection (typically encountered in computational fluid dynamics) will appear when dealing with the momentum balance (transient analysis) and the constitutive equation (transient and quasistatic analysis). Some kind of upwinding is required to avoid the oscillations of the standard Galerkin method. Several approaches can be found in the literature, such as the Petrov-Galerkin method, the Taylor Galerkin method, ${ }^{26}$ the cha

for a general review and techniques have been used

$3,8,22$ and 23 .

3.3. Algorithms for ALE stiess update
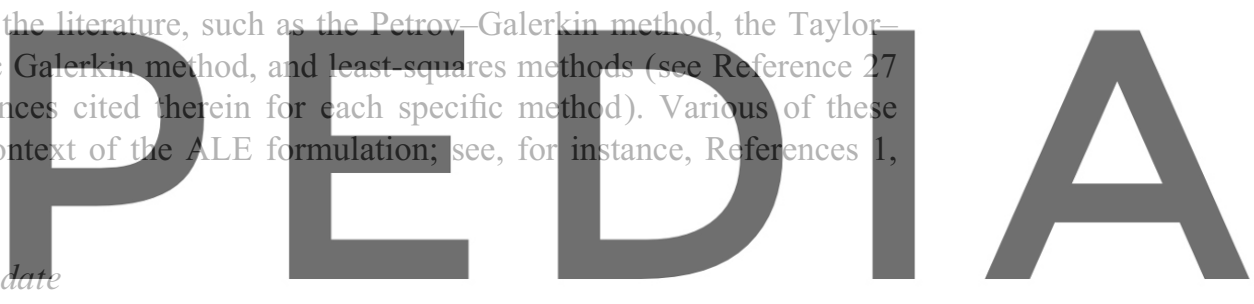

Register for fr fhe material (or Lagrangian) phase

effects are accounted for. The constitutive equation then reads

$$
\left.\frac{\partial \boldsymbol{\sigma}}{\partial t}\right|_{\chi}=\mathbf{q}
$$

and it must be integrated in time to update stresses from ${ }^{n} \boldsymbol{\sigma}$ (stress at time $t_{n}$ ) to ${ }^{\mathrm{L}} \boldsymbol{\sigma}$ (stress after the Lagrangian phase). Neglecting the convective terms is equivalent to assuming that grid points $\chi$ move attached to material particles $\mathbf{X}$. Because of this, the Lagrangian phase can be performed with the same stress update algorithms as used in Lagrangian simulations, which handle the constitutive equation at the Gauss-point level. Various techniques of this type have been employed in this work. All of them preserve the objectivity of the constitutive equation, that is, they treat rigid rotations properly. ${ }^{28,29}$

\subsubsection{The convection (or transport) phase}

The time step is completed with a convection phase, which handles the convective terms not taken into account during the material phase. The constitutive equation is then

$$
\left.\frac{\partial \boldsymbol{\sigma}}{\partial t}\right|_{\chi}+c_{j} \frac{\partial \boldsymbol{\sigma}}{\partial x_{j}}=\mathbf{0}
$$

and its time integration is required to update stresses from ${ }^{\mathrm{L}} \boldsymbol{\sigma}$ to ${ }^{n+1} \boldsymbol{\sigma}$ (i.e. the stress at time $t_{n+1}$ ). 
Remark 3. The fractional-step strategy has resulted in the splitting of the constitutive equation (7) into the parabolic equation (10) of the material phase and the hyperbolic equation (11) of the convection phase. This enables the use of specific algorithms, specially designed to handle each type of equation.

Since it contains a convective term, some type of upwinding is needed to handle equation (11). However, the convective velocity $\mathbf{c}$ is usually moderate (in practice, the mesh velocity $\widehat{\mathbf{v}}$ is often selected by modifying the material velocity $\mathbf{v}$ in order to reduce element distortion), so the convective term is not large. In fact, the main source of trouble in the convective term is the stress gradient, not the convective velocity. As remarked previously, the stress gradient cannot be properly computed at the element level.

A possible solution to this problem is that of Reference 7: in the context of ALE quasistatic analysis, a continuous stress field is obtained by least-squares approximation; the gradient of the smoothed, continuous stress field is then employed in equation (11). The main disadvantage of this approach is that it results in an implicit algorithm, which may be acceptable for quasistatic analyses (although explicit schemes are preferred, as commented previously) but not for transient analyses, where the momentum balance is treated explicitly. More recently, yarious explicit procedures for stress update in ALE transi

In this work, two expl discussed. To avoid comp two different approaches Wendroff update) or (ii) ent (Godunov-like technique)
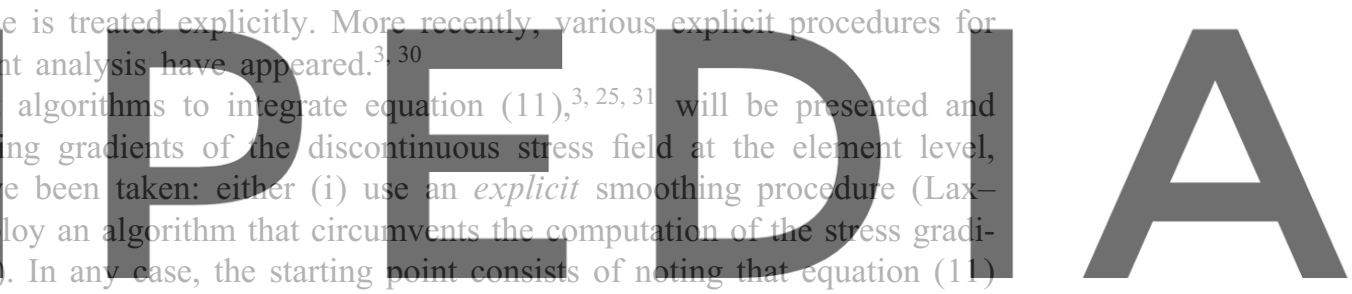

contains a scalar equation for every stress component $\tau$

\section{Register for free at https//www.scipedia.com to download the version without the watermark}

In fact, the internal variables commonly employed in nonlinear mechanics must also be convected following equation (12), so $\tau$ can be any stress-related component (a component of the stress tensor or an internal variable).

Remark 4. The term stress-related component does not imply that only stress measures are employed as internal variables. In isotropic plasticity, for instance, is common to choose the equivalent plastic strain as an internal variable. ${ }^{13}$ Stress-related component is just a convenient way to refer to all the variables that must be updated according to equation (12).

\section{Algorithm 1: Lax-Wendroff update}

As classically done in Lax-Wendroff or Taylor-Galerkin schemes, ${ }^{32,33,26}$ a Taylor series expansion of equation (12) is followed by substitution of the original equation into the expansion. This yields

$$
{ }^{n+1} \tau={ }^{\mathrm{L}} \tau-\Delta t^{n+1 / 2} c_{j} \frac{\partial^{\mathrm{L}} \tau}{\partial x_{j}}+\frac{\Delta t^{2}}{2}{ }^{n+1 / 2} c_{i}{ }^{n+1 / 2} c_{j} \frac{\partial}{\partial x_{i}}\left(\frac{\partial^{\mathrm{L}} \tau}{\partial x_{j}}\right)
$$

where ${ }^{n+1 / 2} \mathbf{c}$ is the convective velocity evaluated at the midstep. It can be checked (see Reference 27) that in this algorithm a correction (with respect to a centred scheme) is 
introduced only in the direction of the convection field, like in streamline-upwind methods. It must be remarked, however, that there is no free or adjustable parameter.

For a transient analysis, ${ }^{n+1 / 2} \mathbf{c}$ is computed directly as the difference of material and mesh velocity,

$$
{ }^{n+1 / 2} \mathbf{c}={ }^{n+1 / 2} \mathbf{v}-{ }^{n+1 / 2} \widehat{\mathbf{v}},
$$

where ${ }^{n+1 / 2} \mathbf{v}$ is obtained in the time integration of the momentum balance and ${ }^{n+1 / 2} \widehat{\mathbf{v}}$ is provided by the ALE remeshing procedure. ${ }^{18,19}$

For a quasistatic analysis, on the other hand, solving the momentum balance yields an increment of material displacements $\Delta \mathbf{u}$ and the ALE remeshing an increment of mesh displacements $\Delta \widehat{\mathbf{u}}$. The convective velocity, which is assumed to be constant within the time step, is then computed as

$$
\mathbf{c}=\frac{\Delta \mathbf{u}-\Delta \widehat{\mathbf{u}}}{\Delta t}
$$

In equation (13), both the stress gradient, which will be denoted by $\gamma\left(\gamma_{i}=\partial^{\mathrm{L}} \tau / \partial x_{i}\right)$, and its spatial derivatives are required. An explicit interpolation procedure, based originally in a classical least-squares projection, point is the integral relation
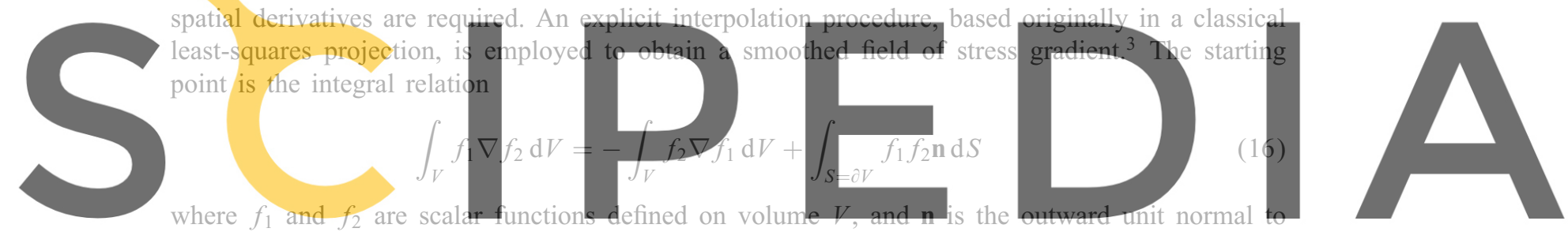

the boundary $S$ of volume $V$. For two-dimensional problems, equation (16) reduces to Register for free at https///www scipedia, com to download the version without the watermark

where $\Omega$ is the two-dimensional domain associated to volume $V$, and $\mathrm{d} \Gamma$ is its boundary. Equation (17) is valid both for plane stress/strain and axisymmetrical problems.

Equation (17) can be specialized into

$$
\int_{R_{x}^{e}} N_{a} \gamma \mathrm{d} V=-\int_{R_{x}^{e}} \tau \nabla N_{a} \mathrm{~d} V+\int_{\partial R_{x}^{e}} N_{a} \tau \mathbf{n} \mathrm{d} S
$$

where $R_{x}^{e}$ and $\partial R_{x}^{e}$ represent element $e$ and its boundary, $\mathbf{n}$ is the outward unit normal in the current configuration and $N_{a}$ is the shape function of node $a$. The assembly of equation (18) results in the linear set of equations

$$
\mathbf{M} \Gamma=\Phi
$$

where $\mathbf{M}$ is a consistent pseudo-mass matrix, $\boldsymbol{\Gamma}$ is a vector of nodal smoothed values of the stress gradient, and the independent vector $\boldsymbol{\Phi}=\left[\boldsymbol{\Phi}_{a}\right]$ is defined as

$$
\boldsymbol{\Phi}_{a}=\sum_{e}\left[-\int_{R_{x}^{e}} \tau \nabla N_{a} \mathrm{~d} V+\int_{\partial R_{x}^{e}} N_{a} \tau \mathbf{n} \mathrm{d} S\right]
$$

To compute the integrals along element boundaries $\partial R_{x}^{e}$, the scalar field $\tau$ is extrapolated from Gauss points to nodes with the aid of Gauss-point shape functions. 
Box 3. Lax-Wendroff stress update

1. Update the mesh configuration from $t_{n}$ to $t_{n+1}$ taking into account the mesh velocity

2. Compute convective velocity $\mathbf{c}$ according to equation (14) (transient processes) or equation (15) (quasistatic processes)

3. Compute the lumped mass matrix $\mathbf{M}^{\mathrm{lp}}$, equation (21)

FOR EVERY STRESS-RELATED COMPONENT $\tau$ :

4. Compute the independent vector $\boldsymbol{\Phi}$, equation (20)

5. Treatment of symmetry: for nodes in an axis/plane of symmetry, set to zero the normal component of $\boldsymbol{\Phi}_{a}$

6. Solve the trivial linear set $\mathbf{M}^{\mathrm{lp}} \boldsymbol{\Gamma}=\boldsymbol{\Phi}$ (diagonal matrix $\mathbf{M}^{\mathrm{lp}}$ ) to get the vector of smoothed nodal gradients $\Gamma$

7. Interpolate stress gradient $\gamma$ and its spatial derivatives from nodes to Gauss points with the aid of shape functions

8. Employ the one-step Lax-Wendroff method to find ${ }^{n+1} \tau$ at the Gauss-point level, equation (22)

Since an explicit procedure is sought, the consistent matrix $\mathbf{M}$ of equation (19) is substituted by the lumped matrix $\mathbf{M}^{\mathrm{lp}}=\left[\mathbf{M}_{a}^{\mathrm{lp}}\right]$, with

$$
\left[\mathbf{M}_{a}^{\mathrm{lp}}\right]=\sum_{e} \int_{R_{x}^{e}} N_{a} \mathrm{~d} V
$$

After doing so, $\boldsymbol{\Gamma}$ is explicitly computed by solving a trivial system of equations, with diagonal matrix $\mathbf{M}^{\mathrm{lp}}$. Then equation (13) can be solved. Since ${ }^{n+1} \tau$ is required at the Gauss points, a collocation technique is used to handle the weak form of equation (13). ${ }^{3}$ This results in

$$
{ }^{n+1} \tau(\xi)={ }^{\mathrm{L}} \tau(\xi)-\Delta t^{n+1 / 2} c_{j}(\xi) \gamma_{j}(\xi)+\frac{\Delta t^{2}}{2}{ }^{n+1 / 2} c_{i}(\xi)^{n+1 / 2} c_{j}(\xi) \frac{\partial \gamma_{j}}{\partial x_{i}}(\xi)
$$

for each Gauss point $\xi$.

A special treatment is required for planes or axes of symmetry. Since the stress-related component $\tau$ is symmetric with respect to an axis or plane of symmetry, the normal component of its gradient $\gamma$ must be set to zero. Since the smoothed nodal gradients are obtained by solving a diagonal set of equations, the cancellation of a certain component $\gamma_{i}$ is equivalent to the cancellation of the corresponding independent term $\Phi_{i}$, prior to the solution of the trivial system. This is the approach taken in the algorithm.

A flowchart of the Lax-Wendroff update technique is presented in Box 3.

\section{Algorithm 2: Godunov-like update}

The second algorithm for the convection phase is based on Godunov's method for conservation laws. ${ }^{34}$ With the help of the stress-velocity product $\mathbf{Y}=\tau \mathbf{c}$ (Reference 8) equation (12) is rewritten as

$$
\left.\frac{\partial \tau}{\partial t}\right|_{\chi}+\frac{\partial Y_{j}}{\partial x_{j}}=\tau \frac{\partial c_{j}}{\partial x_{j}}
$$




\subsubsection{One-point quadratures}

Godunov's method, which was developed in the context of finite volumes, assumes a piecewise constant field $\tau .{ }^{34}$ In a finite element framework, this is the situation if one-point quadratures are employed; Godunov's method is then directly applicable. However, to allow for a subsequent generalization to multiple-point quadratures, a residual weak formulation of the method is preferred. ${ }^{3}$ Since the test functions $\omega$ are also piecewise constant, the integral equation is valid at the element level:

$$
\int_{R_{x}^{e}} \omega \frac{\partial \tau}{\partial t} \mathrm{~d} V=\int_{R_{x}^{e}} \omega \tau \frac{\partial c_{j}}{\partial x_{j}} \mathrm{~d} V-\int_{\partial R_{x}^{e}} \omega(\mathbf{Y} \cdot \mathbf{n}) \mathrm{d} S
$$

Since both $\tau$ and $\omega$ are constant within finite element $e$, equation (23) results in

$$
\frac{\partial \tau}{\partial t}=-\frac{1}{2 V} \sum_{s=1}^{N_{s}} f_{s}\left(\tau_{s}^{\mathrm{c}}-\tau\right)\left[1-\operatorname{sign}\left(f_{s}\right)\right]
$$

where $\tau$ is the stress-related component of element $e$, which has volume $V$ and $N_{s}$ faces, $\tau_{s}^{\mathrm{c}}$ is the stress-related component in the contiguous element across face $s$, and $f_{s}$ is the flux of convective velocity $\mathbf{c}$ across face $s, f_{s}=\int_{s} \mathbf{c} \cdot \mathbf{n} \mathrm{d} S$. Equation (24) corresponds to a full-donor approximation, where full upwinding is achieved by means of $\operatorname{sign}\left(f_{s}\right)$. Contrary to the Lax-Wendroff scheme, however, this upwinding is isotropic and not only along the streamlines. Because of this, the Godunov-like update results in spurious crosswind diffusion and a more overdiffusive behaviour than the Lax-Wendroff update; see Table I in Section 4.1.

For two-dimensional problems, equation (24) can be simplified into

$$
\frac{\partial \tau}{\partial t}=-\frac{1}{2 A} \sum_{\Gamma=1}^{N_{\Gamma}} f_{\Gamma}\left(\tau_{\Gamma}^{\mathrm{c}}-\tau\right)\left[1-\operatorname{sign}\left(f_{\Gamma}\right)\right]
$$

where $N_{\Gamma}$ is the number of edges of element $e$ and $\tau_{\Gamma}^{\mathrm{c}}$ is the stress-related component in the contiguous element across edge $\Gamma$. For plane strain and plane stress problems, $A$ is the area of element $e$ and $f_{\Gamma}$ is the flux across edge $\Gamma, f_{\Gamma}=\int_{\Gamma} \mathbf{c} \cdot \mathbf{n} \mathrm{d} \Gamma$. For axisymmetric problems, on the other hand, axisymmetry is accounted for by taking $A=\int_{R_{x}^{e}} r \mathrm{~d} S$ and $f_{\Gamma}=\int_{\Gamma}(\mathbf{c} \cdot \mathbf{n}) r \mathrm{~d} \Gamma$, where $r$ is the radial co-ordinate.

Explicit time integration of equation (25) yields

$$
{ }^{n+1} \tau={ }^{\mathrm{L}} \tau-\frac{\Delta t}{2 A} \sum_{\Gamma=1}^{N_{\Gamma}} f_{\Gamma}\left(\tau_{\Gamma}^{\mathrm{c}}-\tau\right)\left[1-\operatorname{sign}\left(f_{\Gamma}\right)\right]
$$

for each element.

\subsubsection{Multiple-point quadratures}

Two different strategies have been tested to extend the Godunov-like update to multiple-point quadratures. ${ }^{31,11}$ The first approach is a generalization of the residual formulation of equation (23) which takes into account that the stress-related component $\tau$ and the test function $\omega$ are no longer piecewise constant.

The second approach, which has shown better results than the first one, is an engineering-like extension of the algorithm for piecewise constant fields. The basic idea is to divide every finite element into various subelements, each of them corresponding to the influence domain of a Gauss point. ${ }^{31}$ If, for instance, quadrilaterals with $2 \times 2$ integration points are employed, every element 


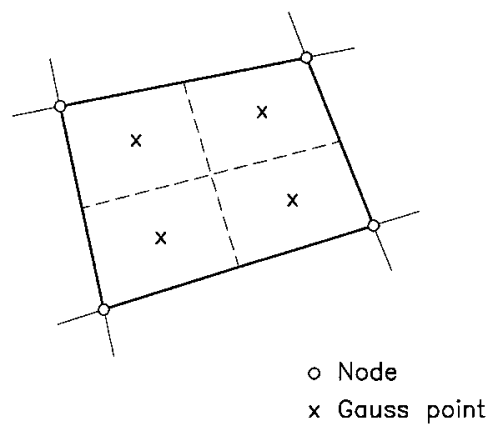

Figure 1. Subelements for the Godunov-like update

Box 4. Godunov-like stress update

1. Update the mesh configuration from $t_{n}$ to $t_{n+1}$ taking into account the mesh velocity

2. Compute convective velocity $\mathbf{c}$ according to equation (14) (transient processes) or equation (15) (quasistatic processes)

3. Transfer convective velocity from original mesh to auxiliary mesh of subelements FOR EVERY SUBELEMENT:

4. Compute area $A$

LOOP ON SIDES OF THE SUBELEMENT

5. Compute the flux of convective velocity across side, $f_{s}$

LOOP ON STRESS-RELATED COMPONENTS

6. Compute jump across side, $\left(\tau_{s}^{\mathrm{c}}-\tau\right)$, and add up the RHS of equation (25)

END OF LOOP ON STRESS-RELATED COMPONENTS

END OF LOOP ON SIDES OF THE SUBELEMENT

7. Employ Godunov's method to find ${ }^{n+1} \tau$ at the subelement level, equation (26)

is divided into four subelements, see Figure 1. In every subelement, $\tau$ is assumed to be constant, and represented by the Gauss-point value. Because of this, $\tau$ is a piecewise constant field with respect to the mesh of subelements, and equation (26) can be employed to update the value of $\tau$ for every subelement. A very simple and efficient algorithm is obtained (see Box 4) because the submeshing of the original mesh into subelements need only be performed once, at the beginning of the analysis.

\subsection{Limitations on the time step}

The numerical time integration of the momentum balance places a limit on the maximum time step $\Delta t$. For transient analysis, this limitation is associated to the stability of the explicit algorithm 
employed to integrate equation ( $5 \mathrm{~b}$ ). For quasistatic analysis, on the other hand, $\Delta t$ is restricted by the convergence properties of the non-linear solver employed for the implicit integration of equation (8).

According to the numerical experimentation in this work, the limitation on time step associated to the numerical time integration of the constitutive equation is not more restrictive than for the momentum balance, in either transient or quasistatic analysis. In other words, the same $\Delta t$ that is used for the momentum balance can then be employed for the stress update, with any of the algorithms of Section 3.3. The basic idea is that, with the typical values of time step $\Delta t$ and convective velocity $\mathbf{c}$, the relative motion between the material and the mesh that occurs during the convection phase carries material particles (at most) to a neighbour element but no further. This situation can be symbolically expressed as

$$
|\mathbf{c}| \Delta t \leqslant h
$$

where $h$ is the element size. In fact, equation (27) is the well-known Courant condition. Since the two algorithms previously presented take into account the stress transport between contiguous elements, they can be used for the stress update in both transient and quasistatic processes.

It must be remarked, then, that the explicit algorithms for the convection phase of Section 3.3 are applicable in combination with either an explicit algorithm for the momentum balance (transient analysis) or an implicit algorithm (quasistatic analysis). In the latter case, there is no need to develop different, implicit stress update procedures for the convection phase. In fact, exactly the same algorithms, without modifications, have been employed for the convection phase of the stress update in the numerical examples of the next section.

If the time integration of the momentum balance is explicit (transient analysis), no iterations are made. Thus, every time step involves performing each of the following tasks once: (1) material phase of the stress update; (2) selection of mesh velocity (i.e., remeshing); (3) convection phase of the stress update. Tasks (2) and (3), which are specific of an ALE analysis and are not present in an UL computation, have a significant impact on the computational cost per time step. It must be recalled, however, that the basic advantage of the ALE formulation it that it avoids element entanglement and keeps element distortion under control. This means that larger time steps than in an UL computation can be used, see Reference 11, and this can have a crucial influence upon the total computational cost.

On the contrary, if the momentum balance is handled by an implicit algorithm (quasistatic analysis), iterations up to equilibrium are required. This means that tasks (1)-(3) must be performed once per iteration, until equilibrium in the ALE mesh is achieved.

An alternative, simplified approach, however, is keeping only task (1) (material phase of the stress update) inside the iteration loop. By doing so, iterations are carried out until equilibrium is verified in the fictitious Lagrangian mesh. After that, tasks (2) and (3) are performed just once, at the end of the time increment (see Box 5). This approach has the advantage that the only overhead cost of ALE quasistatic analysis with respect to an UL analysis is due to the explicit remeshing and the convection phase required at the end of every time step. This overhead cost is negligible when compared to the cost of the implicit time integration of the equilibrium equation. It can be concluded that upgrading a quasistatic analysis from UL to ALE can be done at no (significant) extra computational cost.

It must be noted, on the other hand, that with the simplified approach of Box 5, equilibrium is only achieved in the fictitious Lagrangian mesh, not in the true ALE mesh. Indeed, the convection phase somewhat disrupts equilibrium. Numerical experimentation, however, shows that the spurious 
Box 5. Simplified approach for ALE quasistatic analysis

1. Iteration loop: neglect the convective velocity and solve the equilibrium equation (8) complemented with the Lagrangian constitutive equation (10) iteratively, as in a Lagrangian analysis

2. Remeshing: compute the convective velocity $\mathbf{c}$ and update the mesh from its fictitious Lagrangian configuration to its true ALE configuration

3. Convection: perform the convection phase of the stress update, equation (11), with any of the algorithms of Section 3.3

residual forces associated to the convection phase are small and do not affect the quality of the solution, see the next section and Reference 23.

\section{NUMERICAL EXAMPLES}

Two numerical examples are presented in this section. The first one is a necking test. Various quasistatic simulations are made to show that the explicit algorithms of Section 3.3 can be employed for the convection phase of the stress update, even in the context of the implicit time-stepping. The second numerical example is a coining test. For this test, both quasistatic and transient simulations are performed, to show that the same ALE stress update strategy can be used for both types of analysis.

\subsection{Necking test}

The necking problem is a well-known benchmark test in non-linear solid mechanics. ${ }^{35}$ A cylindrical bar with circular cross-section, with a radius of $6.413 \mathrm{~mm}$ and $53.334 \mathrm{~mm}$ length, is subjected to uniaxial extension. A slight geometric imperfection (1 per cent reduction in radius) induces necking in the central part of the bar. Because of symmetry, only a quarter of the specimen is modelled. The elastoplastic constitutive law can be found in Reference 35. Two meshes of eightnoded quadrilaterals with $2 \times 2$ Gauss points are employed for the analysis, a coarse mesh of 50 elements and a fine mesh of 320 elements. Both UL and ALE simulations have been carried out, for comparison purposes. For the ALE cases, the simplified approach of Box 5 has been employed.

Figure 2 shows the whole deformed piece after an axial elongation of $14 \mathrm{~mm}(7 \mathrm{~mm}$ for half the piece, or 26 per cent of initial length). If the UL formulation is employed in combination with the coarse mesh, Figure 2(a), the elements in the neck zone become very distorted, following the large material deformation. As a consequence, a poor definition of the deformed shape of the piece is obtained.

The quality of the solution may be improved by performing the UL simulation on the fine mesh, Figure 2(b). An alternative approach, however, is to keep the coarse mesh and use the ALE formulation. A very simple ALE remeshing strategy is suggested by the results of the UL analyses: (1) the upper part of the piece, where strains are not large, remains Lagrangian (that is, convective velocity is set to zero, so there is no need to perform the convection phase of the stress update) and (2) equal height of elements is prescribed in the central part, thus avoiding the excessive distortion of elements near the neck of Figure 2(a). The two update algorithms result in very similar deformed shapes; the output with the Lax-Wendroff update is shown in Figure 2(c). 


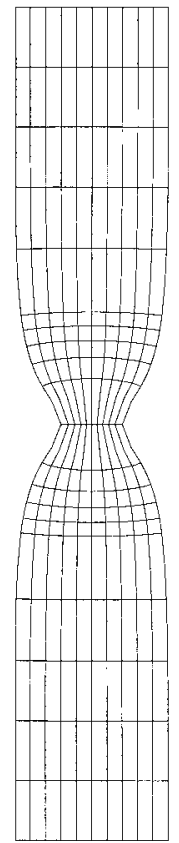

(a)

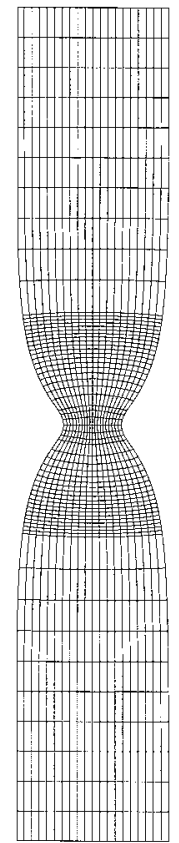

(b)

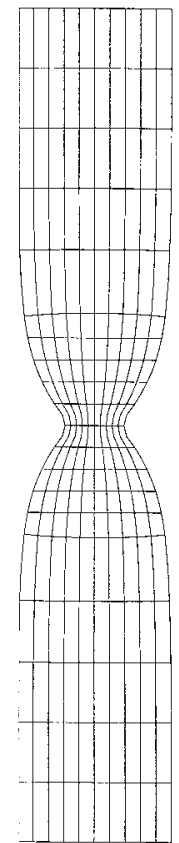

(c)

Figure 2. Necking test. Deformed mesh after 26 per cent elongation: (a) UL formulation with coarse mesh; (b) UL formulation with fine mesh; (c) ALE formulation with fine mesh

A more quantitative comparison of the various simulations is offered in Figure 3, which shows the evolution of radius reduction (ratio of current radius to initial radius) to elongation (in $\mathrm{mm}$, for half the specimen). It can be seen that, up to $7 \mathrm{~mm}$ elongation, all the curves are very similar and in good agreement with those in Reference 35. If pulling proceeds, however, discrepancies arise between the UL solution with the coarse mesh, on one side, and the UL solution with the fine mesh and the ALE solutions, on the other. With only one row of (very distorted) elements in the necking zone, the UL simulation on the coarse mesh does not fully capture the plastification process, and this results in less necking.

A closer look to this behaviour is presented in Figure 4. Taking the Lagrangian solution with the fine mesh as a reference, the relative error in radius of the simulations with the coarse mesh is plotted versus elongation. After a pull of $7 \mathrm{~mm}$, the UL solution offers an acceptable error of 5 per cent, compared to ALE values of slightly under 1 per cent. If pulling continues up to $8 \mathrm{~mm}$, however, the error for the UL solution grows to 80 per cent while it stays below 5 per cent for the two ALE cases, see Figure 4(a). A zoom focusing on the ALE analyses, Figure 4(b), shows that the two update procedures yield almost identical results.

It is apparent from Figures 3 and 4 that the evolution of necking is properly described by the two ALE stress update algorithms, and that tougher criteria must be employed to assess their relative performance. A possibility is to compare the distributions of a certain stress-related component. Plate 1 shows the profiles of the equivalent plastic strain in the neck zone after an elongation of $7 \mathrm{~mm}$. Again, the UL analysis with the fine mesh is taken as a reference, Plate 1(a). 


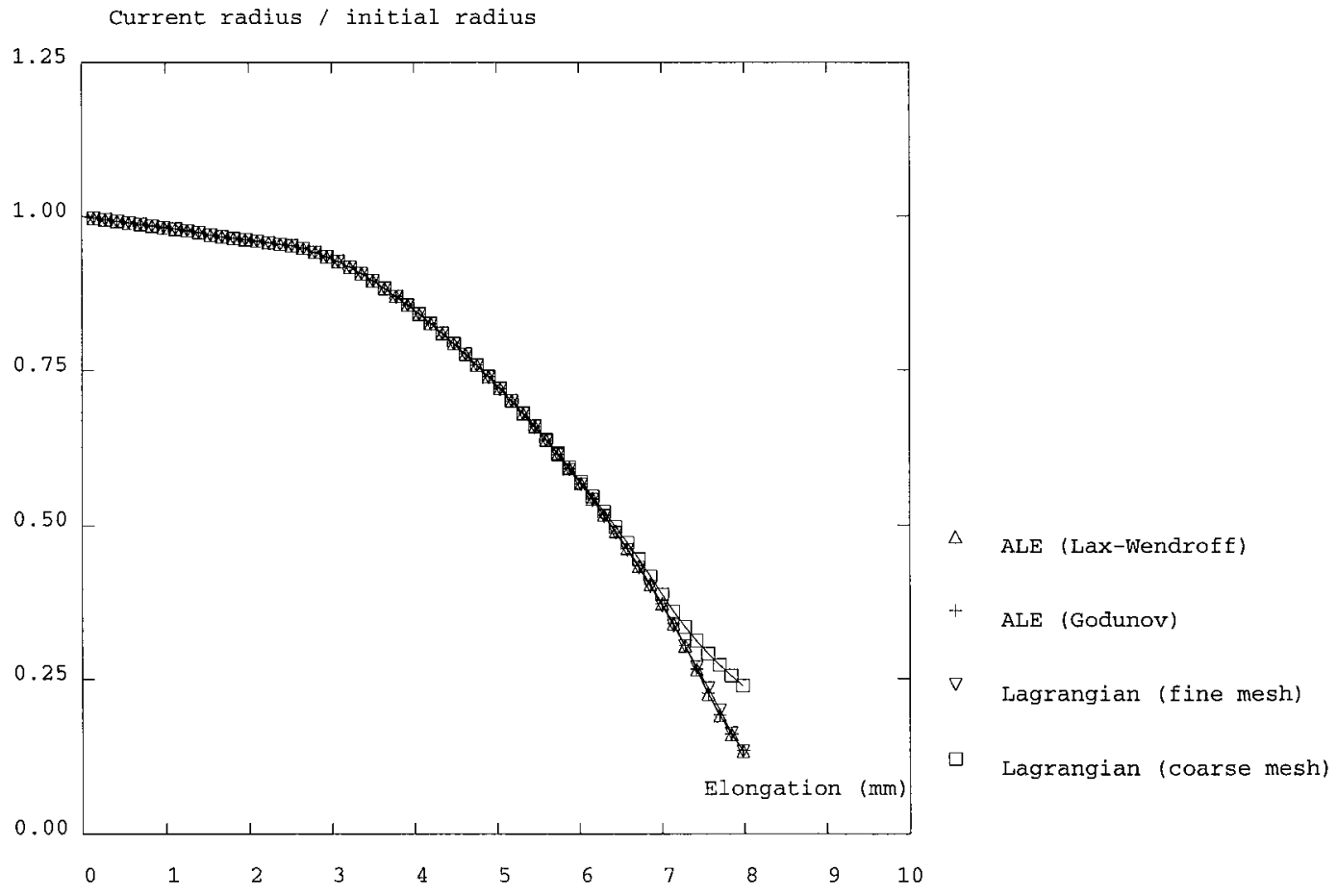

Figure 3. Necking test. Radius reduction vs. bar elongation

The UL simulation with the coarse mesh correctly describes the general aspect of the field, see Plate 1(b), but fails to capture the large plastification in the neck and underestimates the peak value of the plastic strain by 23 per cent, see Table I. As for the ALE cases, the Lax-Wendroff update, Plate 1(c), performs slightly better than the Godunov-like update, Plate 1(d), with an underestimation of the peak value of only 4 and 6 per cent, respectively.

A final, qualitative assessment of the various simulations can be made by looking at the distribution of the Von Mises stress. According to some empirical and semianalytical studies, ${ }^{36}$ this field is constant in the neck zone, along the $z=0$ plane of symmetry. Plate 2 shows the distribution of the Von Mises stress after an elongation of $7 \mathrm{~mm}$. It can be seen that the UL analysis with the coarse mesh, Plate 2(b), does not provide a constant value along $z=0$. On the other hand, both the UL analysis with the fine mesh, Plate 2(a), and the two ALE computations, Plate 2(c) and (d), yield very similar Von Mises stress profiles, which are constant along $z=0$. Again, the suitability of the explicit algorithms of Section 3.3 for the stress update is illustrated with this qualitative comparison.

\subsection{Coining test}

In this test, a coining process is simulated. ${ }^{11}$ A metallic disk, with a radius of $30 \mathrm{~mm}$ and a height of $10 \mathrm{~mm}$, is deformed by a punch $12 \mathrm{~mm}$ in radius, see Figure 5 . The disk is made of an elastoplastic material with elastic modulus $E=200 \mathrm{GPa}$, Poisson's coefficient $v=0 \cdot 3$, yield 
stress $\sigma_{\mathrm{y}}=250 \mathrm{MPa}$ and plastic modulus $E_{\mathrm{p}}=1 \mathrm{GPa}$. Both the punch and the die are rigid. Perfect friction (stick) conditions are assumed in the punch-disk and disk-die interfaces. An axisymmetric analysis is performed to model a 60 per cent height reduction. The finite element mesh is made of $8 \times 20$ eight-noded elements, with $2 \times 2$ Gauss points.

Both quasistatic and transient simulations have been performed, to show that the same explicit algorithms of Section 3.3 can be successfully employed for the convection phase of the stress update in both types of analyses.

Figure 6 shows the output of an UL quasistatic simulation. It can be seen that, at 36 per cent height reduction, the finite elements under the punch corner and in contact with the die become very distorted, and the analysis cannot proceed. The pattern of material flow is also clear from Figure 6: since there is perfect friction in the two interfaces, the material tends to flow outward from the central part of the disk. The outer part of the disk is relatively unaffected by deformation and moves in a rather rigid manner.

With the ALE description, on the contrary, the analysis can be completed. Plate 3 shows the evolution of the ALE mesh and the yield stress. The Godunov-like algorithm is employed for the convection phase of the ALE stress update. A similar result is obtained with the Lax-Wendroff algorithm.

To assess the influence of dynamic effects, various ALE transient simulations of this coining process have been performed, with punch velocities $\mathbf{v}$ ranging from 300 to $0.15 \mathrm{~m} / \mathrm{s}$. Plate 4 shows the final ALE mesh and yield stress profile for different values of v. As in the quasistatic case,

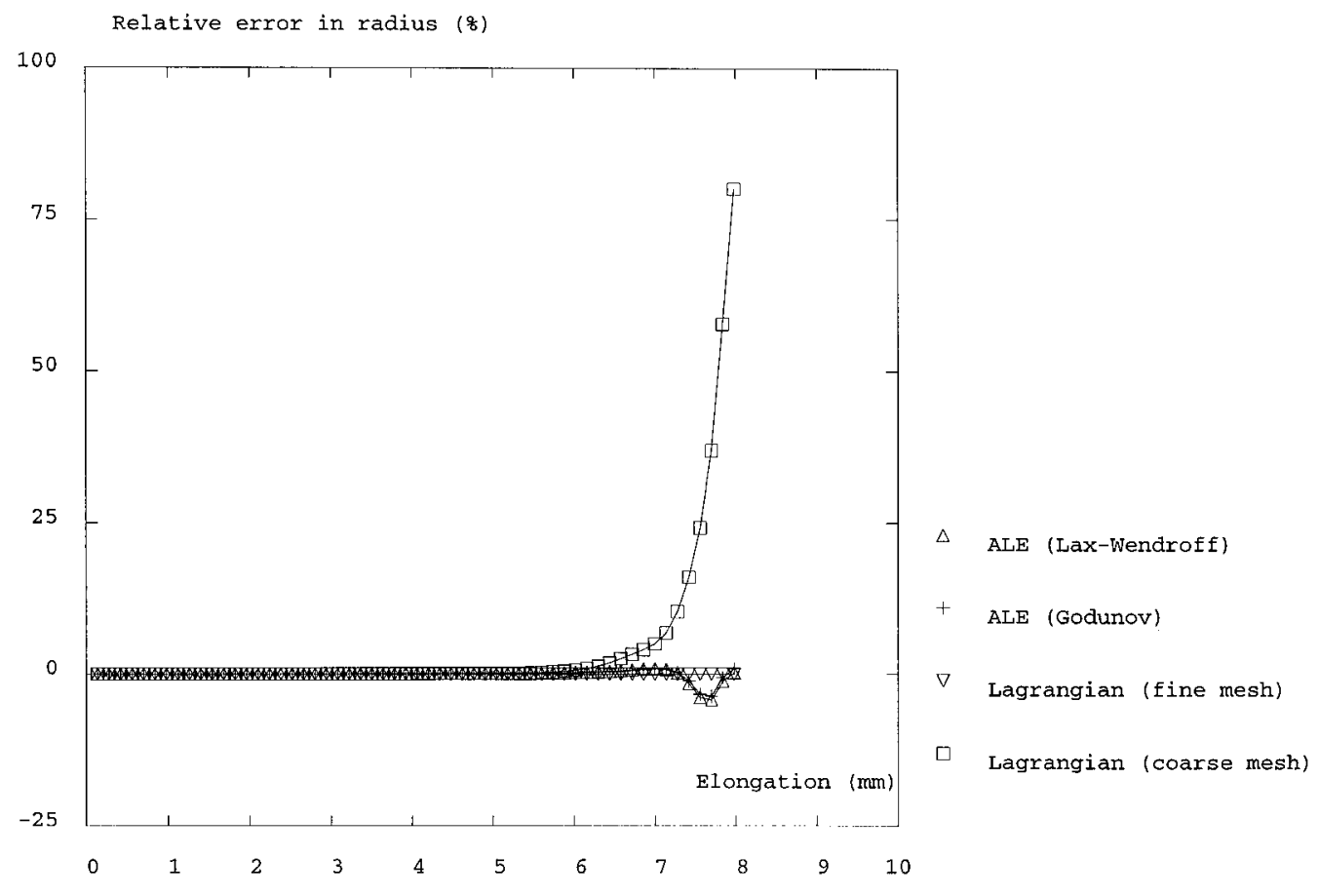

Figure 4(a). Necking test. Relative error in radius reduction vs. bar elongation; full view 
ALE STRESS UPDATE

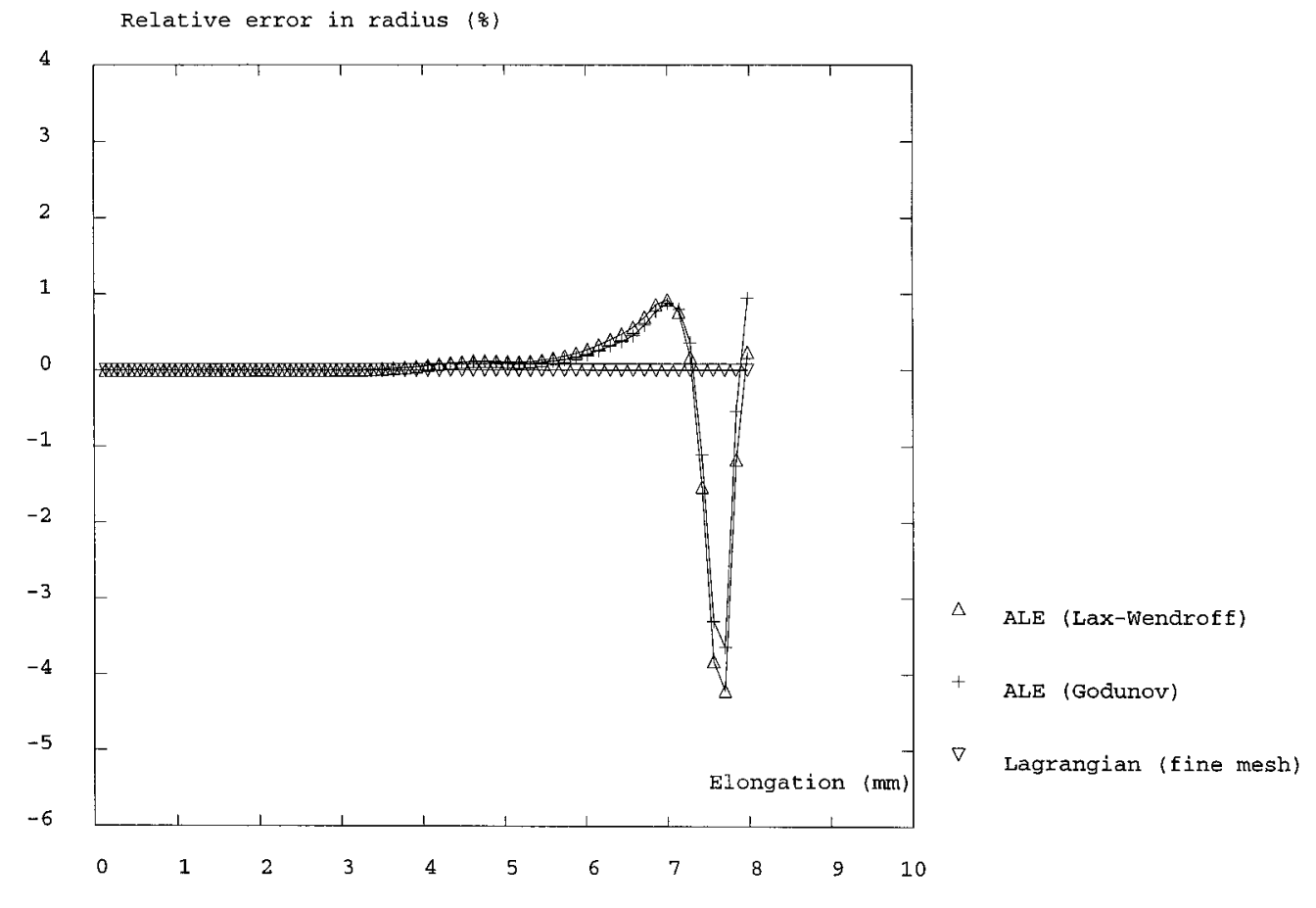

Figure 4(b). Necking test. Relative error in radius reduction vs. bar elongation; zoom

Table I. Necking test. Maximum plastic strain for various simulations

\begin{tabular}{lcc}
\hline & Max. plastic strain & Relative error \\
\hline $\begin{array}{l}\text { Lagrangian analysis } \\
\text { Fine mesh }\end{array}$ & $2 \cdot 28$ & Reference value \\
Coarse mesh & 1.76 & $-23 \%$ \\
& & \\
ALE analysis, coarse mesh & & $-4 \%$ \\
$\quad$ Lax-Wendroff update & $2 \cdot 18$ & $-6 \%$ \\
Godunov update & $2 \cdot 14$ & \\
\hline
\end{tabular}

similar results are obtained with the two explicit algorithms for the stress update; the Lax-Wendroff update is employed in Plate 4.

It is interesting to note that the output of the quasistatic and transient analyses are in good agreement, in the sense that the last picture of Plate 3 (quasistatic analysis) is the limit case with null inertia effects of the sequence of punch velocities of Plate 4 (transient analysis), both in terms of deformed shape and yield stress profile. This result corroborates the applicability of the explicit algorithms to both ALE quasistatic and transient analysis, in spite of the inherent differences in the time-marching strategy (implicit vs. explicit). 
A. RODRÍGUEZ-FERRAN, F. CASADEI AND A. HUERTA

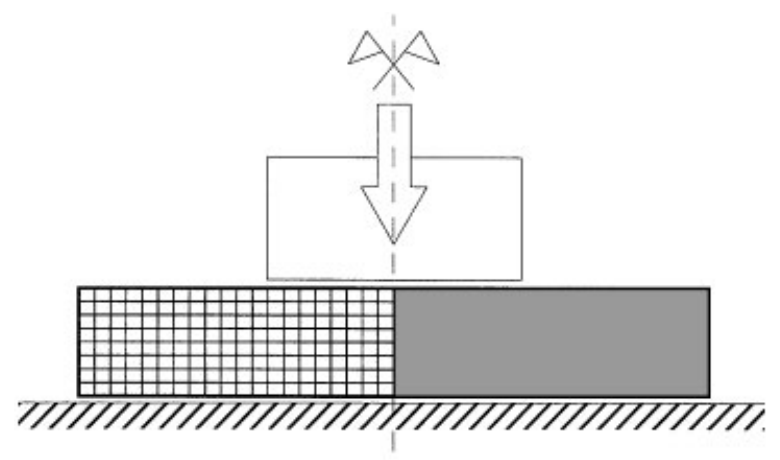

Figure 5. Coining test. Problem statement

08

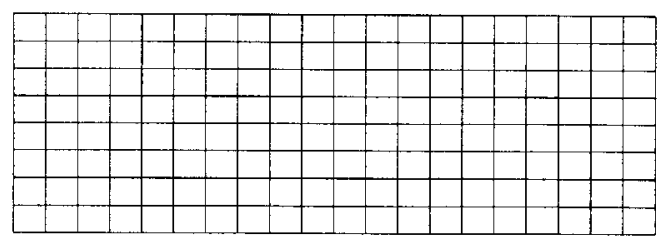

128
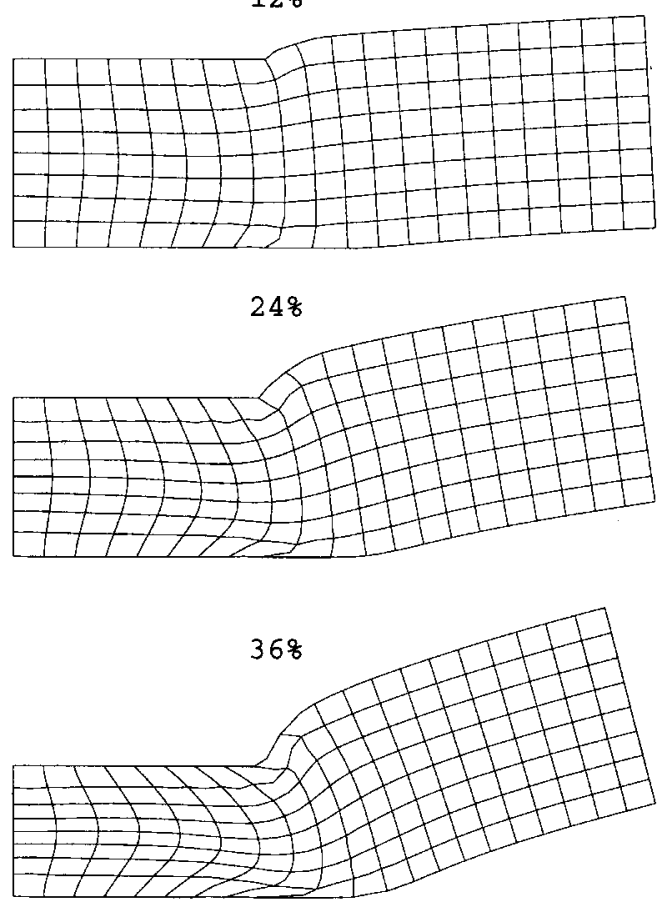

Figure 6. Coining test, quasistatic analysis. Distorted mesh in an UL computation 


\section{CONCLUDING REMARKS}

A unified presentation of the Arbitrary Lagrangian-Eulerian (ALE) formulation for quasistatic and transient analysis has been made. In the general (i.e. transient) case, the governing equations have convective terms that reflect the relative motion between mesh and material. In the quasistatic case, on the contrary, there are no convective terms in the momentum balance (because inertia effects associated to the convective acceleration are neglected), but they are still present in the constitutive equation (representing the arbitrary mesh motion which is inherent to ALE kinematics).

Numerical time-integration of the governing equations has been performed by means of fractional-step methods, thus treating material and convection terms in two separate phases. It has been shown that the same explicit algorithms (Lax-Wendroff and Godunov-like) can be employed for the convection phase of the stress update for both quasistatic and transient problems, in spite of the different time-marching strategy (implicit for quasistatic, explicit for transient). This means that there is no need to develop specific algorithms for each type of computation. The most important consequence, however, is that a quasistatic simulation can be upgraded from Updated Lagrangian to ALE without a significant computational overhead, because the additional features of the ALE analysis (convection phase and remeshing) are explicit, while the time-integration of the equilibrium equation is implicit. These ideas are illustrated by means of two numerical examples in nonlinear solid mechanics, a necking test and a coining test.

\section{REFERENCES}

1. J. Donea, 'Arbitrary Lagrangian-Eulerian finite element methods', in T. Belytschko and T. J. R. Hughes (eds.), Computational Methods for Transient Analysis, Chapter 10, Elsevier, New York, USA, 1983.

2. S. Giuliani, 'An algorithm for continuous rezoning of the hydrodynamic grid in arbitrary Lagrangian-Eulerian computer codes', Nucl. Engng. Des., 72, 205-212 (1982).

3. A. Huerta and F. Casadei, 'Arbitrary Lagrangian-Eulerian formulation for large boundary motion in nonlinear continuum mechanics', Technical Note No. 1.91.95, Safety Technology Institute, Joint Research Centre Ispra, European Commission. Also in Internal Report MA006/1991, E.T.S. de Ingenieros de Caminos, Universitat Politècnica de Catalunya, 1991.

4. J. P. Ponthot, 'Traitement Unifié de la Mécanique des Milieux Continus Solides en Grandes Transformations par la Méthode des Éléments Finis', Doctoral Thesis, University of Liège, 1995 (in French).

5. J. Sarrate, 'Modelización numérica de la interacción fluido-sólido rígido: desarrollo de algoritmos, generación de mallas y adaptabilidad', Doctoral Thesis, E.T.S. de Ingenieros de Caminos, Universitat Politècnica de Catalunya, Barcelona, 1996, (in Spanish).

6. P. Schreurs, 'Numerical simulation of forming processes. The use of the arbitrary-Eulerian-Lagrangian (AEL) formulation and the finite element method', Dissertation, University of Eindhoven, The Netherlands, 1983.

7. J. Huétink, 'On the simulation of thermo-mechanical forming processes', Dissertation, University of Twente, The Netherlands, 1986.

8. W. K. Liu, T. Belytschko and H. Chang, 'An arbitrary Lagrangian-Eulerian finite element method for path-dependent materials', Comput. Meth. Appl. Mech. Engng., 58, 227-245 (1986).

9. D. J. Benson, 'An efficient, accurate, simple ALE method for nonlinear finite element programs', Comput. Meth. Appl. Mech. Engng., 72, 305-350 (1989).

10. S. Ghosh and N. Kikuchi, 'An arbitrary Lagrangian-Eulerian finite element method for large deformation analysis of elastic-viscoplastic solids', Comput. Meth. Appl. Mech. Engng., 86, 127-188 (1991).

11. F. Casadei, J. Donea and A. Huerta, 'Arbitrary Lagrangian-Eulerian finite elements in non-linear fast transient continuum mechanics', Report EUR 16327 EN, Institute for Safety Technology, Joint Research Centre Ispra, European Commission, 1995.

12. A. Huerta and F. Casadei, 'New ALE applications in non-linear fast-transient solid dynamics', Engng. Comput., 11, 317-145 (1994).

13. A. S. Khan and S. Huang, Continuum Theory of Plasticity, Wiley, New York, 1995.

14. A. Huerta and W. K. Liu, 'Large amplitude sloshing with submerged blocks', J. Pressure Vessel Technol. ASME, 112, 104-108 (1990).

15. L. W. Malvern, Introduction to the Mechanics of a Continuous Medium, Prentice-Hall Series in Engineering of the Physical Sciences, Englewood Cliffs, N.J., USA, 1969. 
16. A. Huerta and W. K. Liu, 'Viscous flow with large free surface motion', Comput. Meth. Appl. Mech. Engng., 69, 277-324 (1988).

17. NUMIFORM, 'Simulation of materials processing: theory, methods and applications', Proc. 5th Int. Conf. on Numerical Methods in Industrial Forming Processes, Ithaca, New York, 1995.

18. J. P. Halleux and F. Casadei, 'Transient large-strain finite element analysis of solids', in C. Taylor, D. R. J. Owen and E. Hinton (eds.), Computational Methods for Non-Linear Problems, Pineridge, Swansea, 1987.

19. G. Pijaudier-Cabot, L. Bodé and A. Huerta, 'Arbitrary Lagrangian-Eulerian finite element analysis of strain localization in transient problems', Int. J. Numer. Meth. Engng., 38, 4171-4191 (1995).

20. D. J. Benson, 'Computational methods in Lagrangian and Eulerian hydrocodes', Comput. Meth. Appl. Mech. Engng., 99, 235-394 (1992).

21. J. Peraire, M. Vahdati, K. Morgan and O. C. Zienkiewicz, 'Adaptive remeshing for compressible flow computations', J. Comput. Phys., 72, 449-466 (1991).

22. W. K. Liu, H. Chang, J. Chen and T. Belytschko, 'Arbitrary Lagrangian-Eulerian Petrov-Galerkin finite elements for nonlinear continua', Comput. Meth. Appl. Mech. Engng., 68, 259-310 (1988).

23. F. P. T. Baaijens, 'An U-ALE formulation of 3-D unsteady viscoelastic flow', Int. J. Numer. Meth. Engng., 36, $1115-1143$ (1993).

24. R. D. Richtmyer and K. W. Morton, Difference Methods for Initial-Value Problems, Interscience, New York, 1967.

25. A. Rodríguez-Ferran and A. Huerta, 'ALE quasistatic analysis in an object-oriented code', Proc. 4th Int. Conf. on Computational Plasticity (COMPLAS IV), Barcelona, Vol. 2, 1995, pp. 2349-2360.

26. J. Donea, 'A Taylor-Galerkin method for convective transport problems', Int. J. Numer. Meth. Engng., 20, 101-120 (1984).

27. J. Donea and L. Quartapelle, 'An introduction to finite element methods for transient advection problems', Comput. Meth. Appl. Mech. Engng., 95, 169-203 (1992).

28. A. Rodríguez-Ferran and A. Huerta, 'Comparing two algorithms to add large strains to a small strain finite element code', Research Report No. 91, International Centre for Numerical Methods in Engineering, Barcelona, 1996.

29. A. Rodríguez-Ferran, P. Pegon and A. Huerta, 'Two stress update algorithms for large strain solid mechanics: accuracy analysis and numerical implementation', Int. J. Numer. Meth. Engng., 40, 4363-4404 (1997).

30. R. Akkerman, J. Huétink, P. N. van der Helm, 'Finite elements and volumes in a Euler-Lagrange formulation. Artificial dissipation versus limited flux schemes', Proc. 4th Int. Conf. on Computational Plasticity (COMPLAS IV), Barcelona, Vol. 2, 1995, pp. 2307-2318.

31. A. Huerta, F. Casadei and J. Donea, 'ALE stress update in transient plasticity problems', Proc. 4th Int. Conf. on Computational Plasticity (COMPLAS IV), Barcelona, Vol. 2, 1995, pp. 1865-1876.

32. P. D. Lax and B. Wendroff, 'Systems of conservations laws', Commun. Pure Appl. Math., 13, $217-237$ (1960).

33. P. D. Lax and B. Wendroff, 'Difference schemes for hyperbolic equations with high-order of accuracy', Commun. Pure Appl. Math., 17, 381 (1964).

34. R. J. Le Veque, Numerical Methods for Conservation Laws, Lectures in Mathematics, ETH Zürich, Birkhäuser Verlag, Basel, 1990.

35. J. C. Simo, 'A framework for finite strain elastoplasticity based on maximum plastic dissipation and the multiplicative decomposition. Part II: computational aspects', Comput. Meth. Appl. Mech. Engng., 68, 1-31 (1988).

36. J. M. Goicolea, 'Numerical modelling in large strain plasticity with application to tube collapse analysis', Ph.D. Thesis, University of London, 1985. 


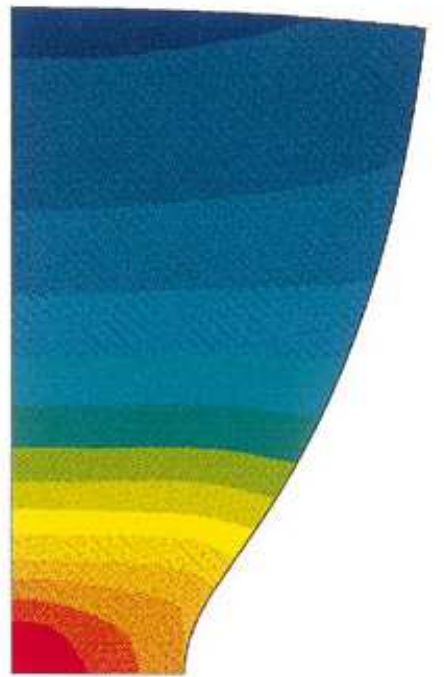

(a)

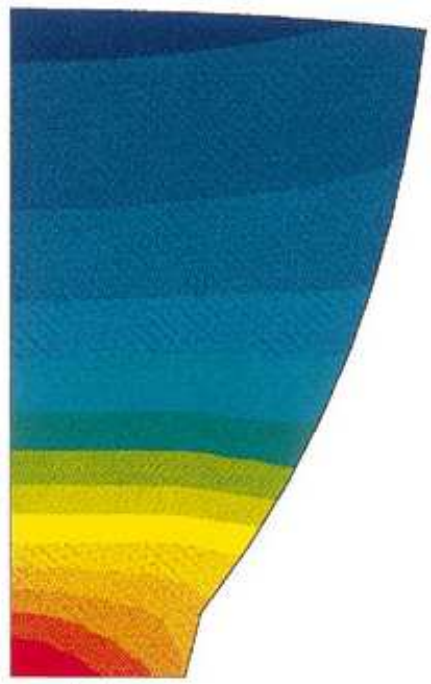

(c)

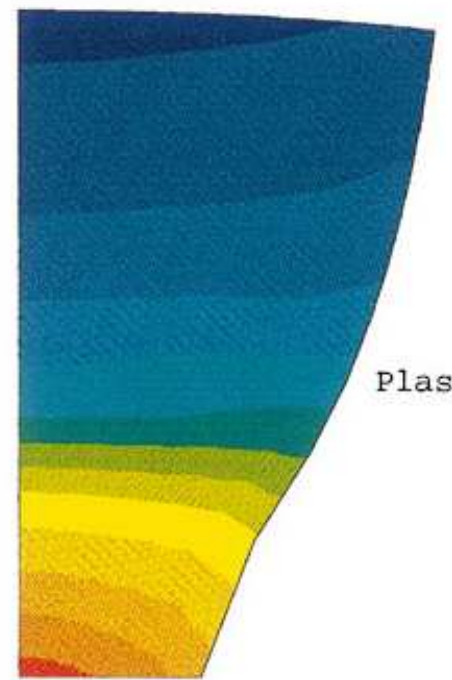

(b)

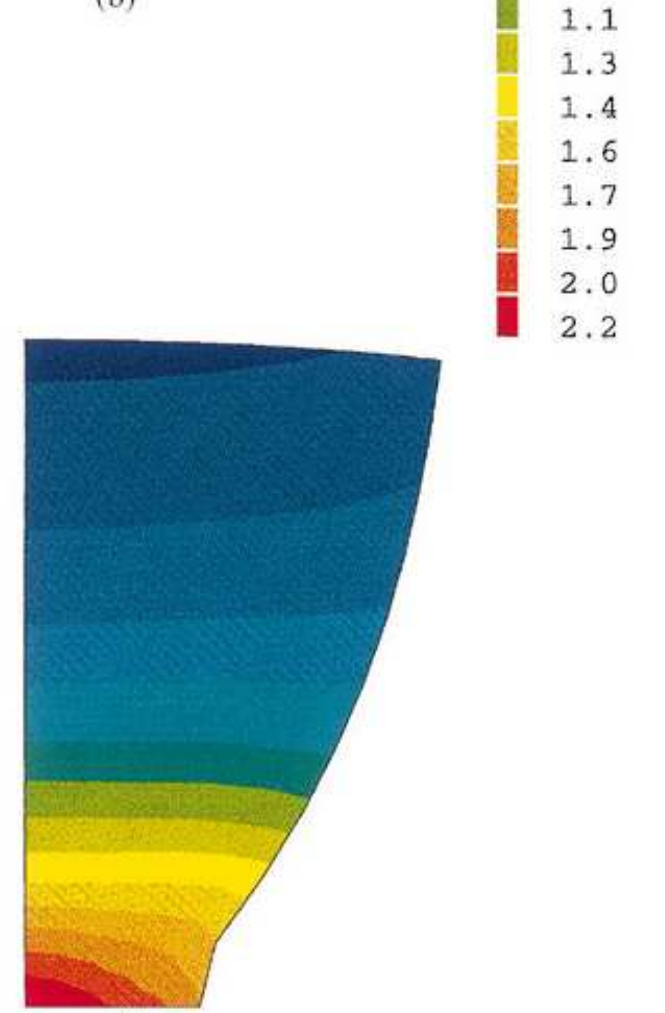

(d)

Plate 1. Necking test. Equivalent plastic strain in the neck zone. UL formulation: (a) fine mesh; (b) coarse mesh. ALE formulation (coarse mesh): (c) Lax-Wendroff update; (d) Godunov-like update 


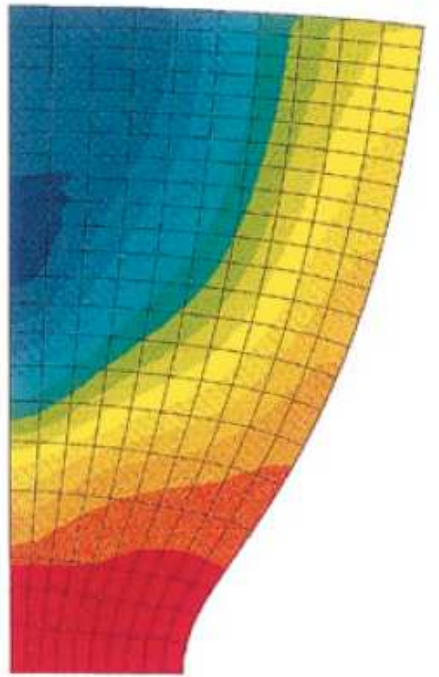

(a)

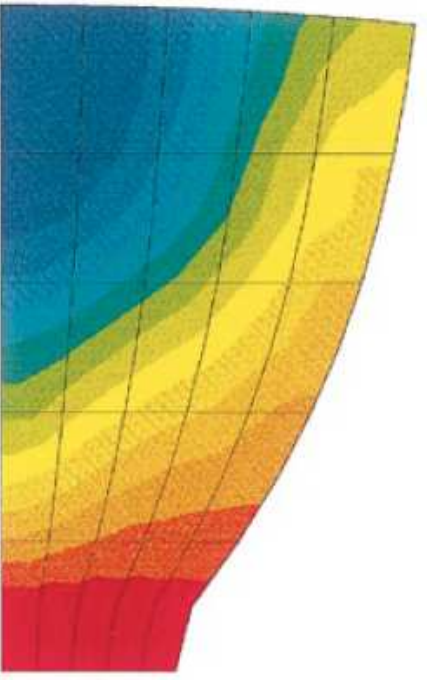

(c)

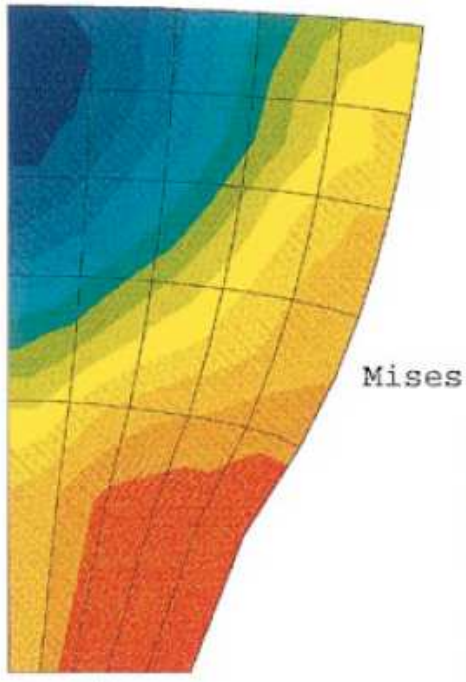

(b)

0.04
0.11
0.18
0.25
0.32
0.39
0.46

0.53

0.60

0.67

0.74

0.81

0.88

0.95

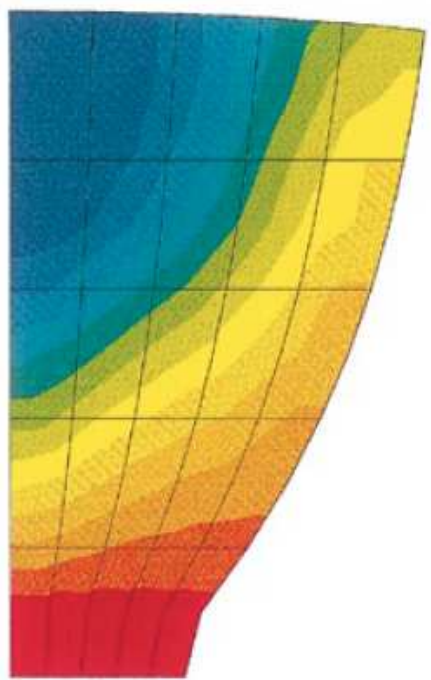

(d)

Plate 2. Necking test. Von Mises stress in the neck zone. UL formulation: (a) fine mesh; (b) coarse mesh. ALE formulation (coarse mesh): (c) Lax-Wendroff update; (d) Godunov-like update 

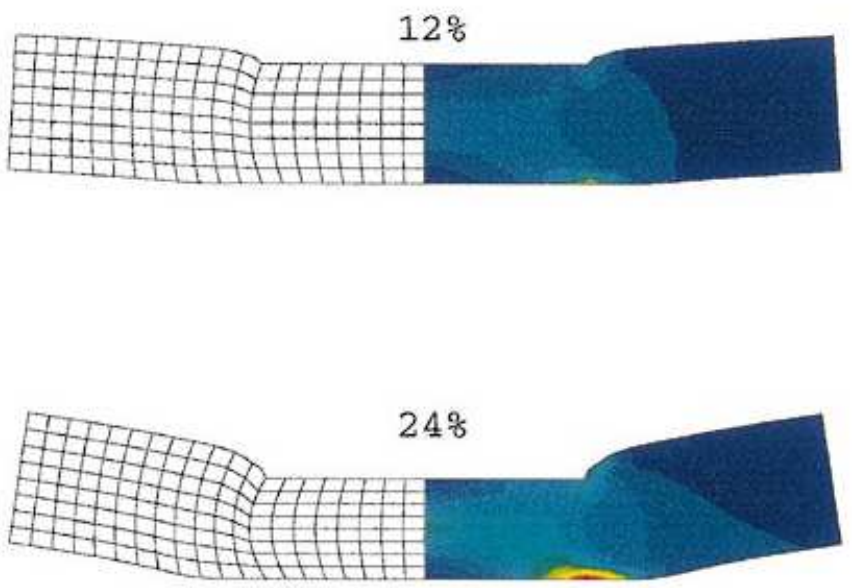

Yield stress (Mpa)
250
350
450
550
650
750
850
950
1050
1150
1250
1350
1450
1550
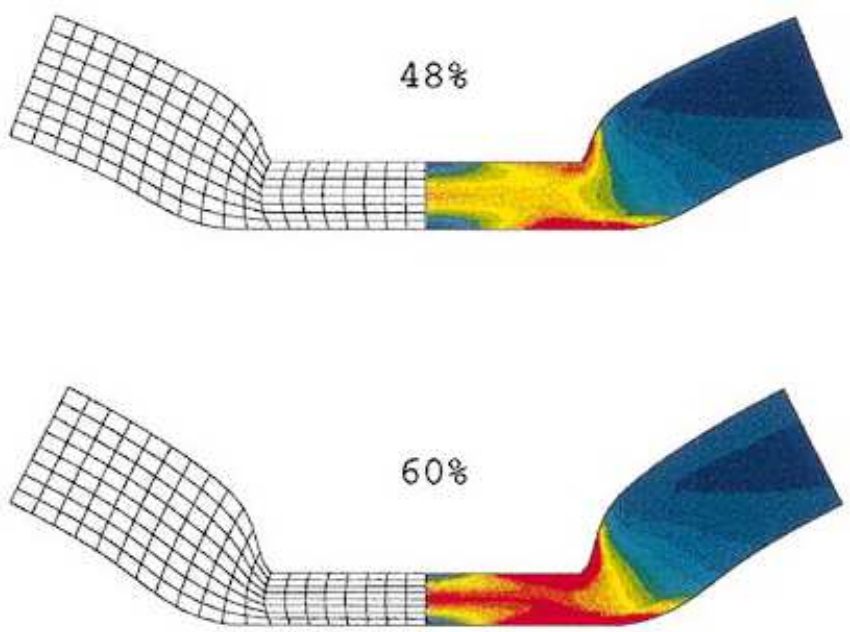

Plate 3. Coining test, quasistatic analysis. Evolution of the ALE mesh and yield stress 


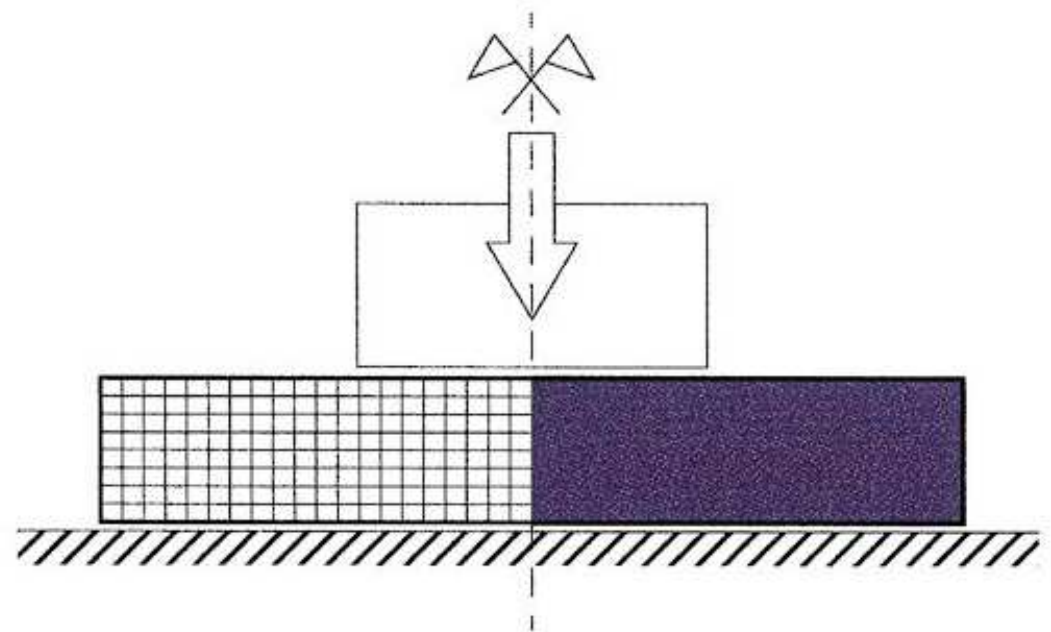

Yield stress (MPa)

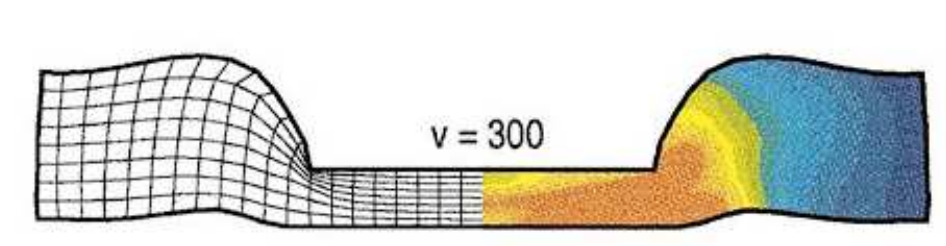

250

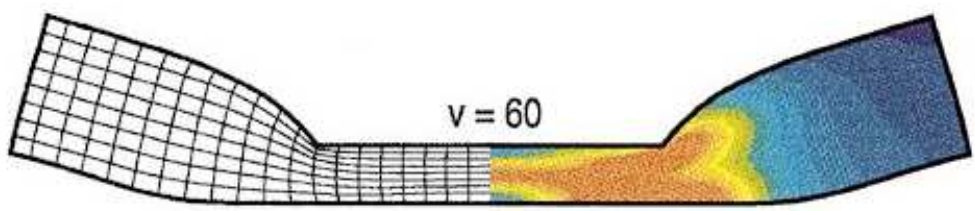

1050

1150

1250

1350

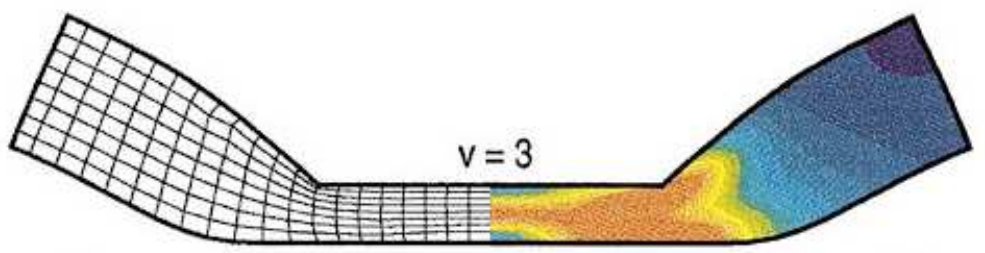

1450

1550

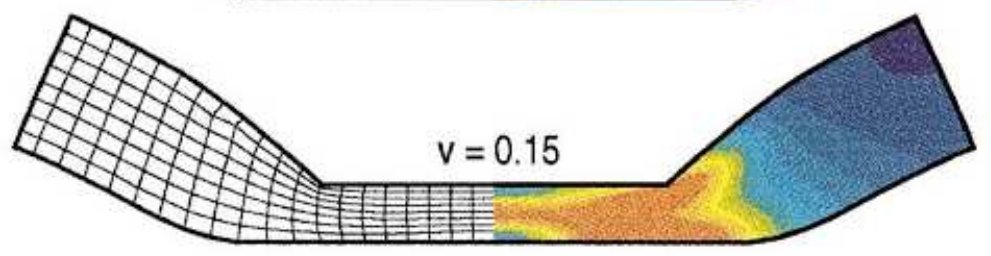

Plate 4. Coining test, transient analysis. Final ALE mesh and yield stress for various punch velocities $v$ 